\title{
Apoyo Social y Adhesión al Tratamiento Antirretroviral en Pacientes VIH+ en un Programa de Mantenimiento con Metadona. Un estudio Longitudinal
}

\section{Social Support and Adhesion to Anti Retroviral Treatment in HIV+ Patients on a Methadone Maintenance Program. A Longitudinal Study}

\author{
Lucía Ladero \\ CAID Torrejón de Ardoz
}

\author{
Santos Orejudo \\ Universidad de Zaragoza
}

\author{
José Antonio Carrobles \\ Universidad Autónoma de Madrid
}

Resumen. Este estudio longitudinal tiene como objetivo analizar las relaciones existentes entre la variable apoyo social y la adhesión a los tratamientos antirretrovirales. Se presenta la evolución de una muestra de 100 pacientes VIH+ en programa de metadona. En el seguimiento, un año después de la primera evaluación, se obtienen los datos de 83 participantes que respondieron al cuestionario y que fueron clasificados en función de su adhesión a la terapia antirretroviral según indicadores del día, de la semana y del mes. En el estudio se comprueba que existen diferencias en la variable considerada entre pacientes con buena adhesión, los que no presentan adhesión, los que inician tratamiento y aquellos que lo abandonan, llegando a discriminar entre estos últimos. Se concluye que el papel del apoyo social resulta muy importante tanto en el inicio, como en el mantenimiento de la adhesión una vez establecida, cumpliendo un papel relevante también, en la pérdida de la misma.

Palabras clave: adhesión, TARGA, apoyo social, drogodependientes, programa de mantenimiento con metadona.

\begin{abstract}
The present study examined the dynamics of IDUs social support and adherence to treatment regimens over time. The participants were $100 \mathrm{HIV}+$ patients on a methadone maintenance program in Madrid (Spain). The data were elicited using a structured, interviewer-administered questionnaire and were followed for one year. We investigate the following 4 groups of patients: those who continued the treatment, those who refused the treatment, those who started the treatment and the patients where there was treatment failure. Conclusions: social support is an important variable in the start, continuation and also in ultimate failure of medical treatment.
\end{abstract}

Key words: adherence, HAART, social support, drug addict, methadone maintenance program. 


\section{Introducción}

En la actualidad existen tratamientos de probada eficacia para los pacientes infectados por el VIH/SIDA. No obstante, la generalización de estos tratamientos en los países más avanzados se ha visto limitada por varios factores, entre los que destaca la necesidad de contar con la colaboración del paciente para cumplimentar las pautas farmacológicas prescritas durante largos periodos de tiempo, de ahí la importancia de la adhesión (Jonson et al., 2006).

En España los usuarios de drogas inyectables continúan siendo la población que se ha visto más afectada por la infección VIH/SIDA. La aparición de los tratamientos para la adicción como puede ser la metadona, junto con la llegada de la Terapia Antirretroviral de Gran Actividad (TARGA) han constituido un avance clínico muy importante, contribuyendo a la esperanza de vida de esta población (Clarke et al., 2003). Aunque está comprobado que la efectividad del tratamiento con metadona mejora cuando existe una intervención psicosocial (Scherbaum, 2005) son muchos los impedimentos que surgen en la aplicación del mismo: falta de compromiso, resistencias, recaídas, etc.; que dificultan asimismo, la adhesión a la TARGA. (Ballester, 2002; Vlahov y Celentano, 2006).

En este mismo sentido se podría hablar de mejores resultados terapéuticos en los tratamientos para drogodependientes en aquellos pacientes que se sienten más apoyados tanto desde sus propias familias, como desde los técnicos sanitarios (Préau et al., 2006; Ballester, 2003), que tienen mejor acceso a los centros de drogas para iniciar tratamiento, o para trabajar la recaída (Skeie et al., 2007; Chen, 2006). Algunos estudios muestran la relación entre el aumento de las conductas de riesgo en la inyección de drogas en aquellos pacientes que se relacionan sólo con consumidores de drogas en activo, mientras que en los pacientes que han cambiado su red social y se relacionan con personas no consumidoras disminuyen estas conductas de riesgo (Costenbader et al., 2006). También, los trabajos llevados a cabo con el grupo de iguales han mostrado buenos resultados en la consecución de una mejor retención y un menor consumo de drogas en población drogodependiente (Skeie et al., 2007).
La mayoría de los estudios realizados con población drogodependiente obtienen como resultado que el consumo de drogas tiene una influencia negativa en la adhesión a la TARGA (Battaglioli- DeNero, 2007; Hinkin et al., 2007) y en algunas investigaciones realizadas sobre la entrevista médica se observa que se recomienda incluso la no prescripción de fármacos antirretrovirales si los pacientes están consumiendo de forma activa (Maisels et al., 2001). Resulta difícil, no obstante, determinar cuáles son las variables que influyen en la falta de adhesión, quizá por las peculiaridades que estos pacientes presentan (problemas sociales, trastornos mentales, dificultades de acceso a recursos, etc).

Las relaciones entre variables de tipo socioeconómicas y la adhesión a los tratamientos antirretrovirales han sido analizadas en algunos estudios con población drogodependiente. Distintos autores han constatado la importancia de variables sociales como el nivel cultural (Reynolds et al., 2004), la edad (Goodkin et al., 2004), el estar empleado (Carballo et al., 2004), no vivir solo, el encarcelamiento (Kerr et al., 2005) con la adhesión a la TARGA.

Se observan mejores resultados en la adhesión a la TARGA en aquellos pacientes que no encuentran barreras en el acceso a las clínicas, que tienen buena comunicación con los sanitarios que los atienden y que se sienten más apoyados emocionalmente tanto para iniciar el tratamiento antirretroviral, como para continuar con el mismo (Knowlton et al., 2006; Shelton et al., 2006).

En población no drogodependiente se observa que mejora la adhesión a la TARGA cuando los pacientes se encuentran más apoyados (Carrobles, Remor y Rodríguez-Alzamora, 2003; Remor, 2002; Waddell et al., 2006;). En general, se entiende demostrada la relación entre el sentirse apoyado y los mejores resultados en la forma de enfrentarse a cualquier enfermedad sea aguda o crónica, debido quizá a que el apoyo social funcionaría como amortiguador de los estados de ánimo negativos (Gustafsson et al., 2006). Asimismo, se observa un aumento en el uso y abuso de sustancias de aquellas personas que se enfrentan solos a sus problemas y que no se sienten apoyadas por su entorno más inmediato (Garrity et al., 2006), o que sus diferen- 
cias económicas, sociales y de género pueden determinar el inicio del consumo de drogas, el mantenimiento o el abandono (Dannerbeck et al., 2006; Mino et al., 2006).

Hasta este momento la mayor parte de las investigaciones sobre la adhesión se han hecho con diseños transversales y retrospectivos siendo los estudios longitudinales menos frecuentes o limitados a espacios temporales breves en los que se busca la evolución de la propia adhesión. No obstante, esta metodología presenta algunas limitaciones para establecer relaciones entre la adhesión y posibles variables antecedentes o consecuentes. Además, la estrategia más habitual de considerar la adhesión en función del número de comprimidos ingeridos o de sujetos que presentan buena o mala adhesión no recoge la complejidad asociada a esta situación (Spire et al., 2002), con cambios en función de los momentos y la descripción de distintos patrones de adhesión, asociados también a distintas razones, contextos, conductas o niveles de apoyo social (Hill et al., 2003).

Con todo esto, el objetivo principal de este trabajo consiste en analizar las relaciones y variaciones experimentadas a lo largo de un año entre la adhesión al tratamiento antirretroviral y el apoyo social en una población en programa de mantenimiento con metadona. Un soporte empírico a la relación entre esta variable y la adhesión aumentaría las posibilidades de intervención para mejorar la eficacia de la TARGA.

\section{Método}

\section{Participantes}

Los sujetos se seleccionaron en dos Centros de Atención Integral a Drogodependencias (CAID) de la Comunidad de Madrid. De los 250 usuarios del centro de Torrejón de Ardoz se seleccionaron los 83 pacientes que cumplían los criterios de inclusión en la investigación (ser mayores de 18 años, portadores de VIH, estar en programa de mantenimiento con metadona y tener una prescripción facultativa de antirretrovirales) y ninguna de las condiciones de exclusión (no aceptar el consentimiento informado, no presentarse físicamente a la cita con el investigador, encontrarse durante el periodo de recogida de datos hospitalizado, encamado o preso). La muestra se completó con otros 17 participantes del CAID de San Blas que cumplían con los criterios señalados anteriormente, $\mathrm{y}$, que fueron seleccionados aleatoriamente, entrando a formar parte de la muestra aquellos que mostraban su consentimiento según pasaban a recoger las dosis de metadona el día elegido.

La muestra está compuesta en su mayoría por hombres $(80 \%)$, de nivel socioeconómico bajo (71\%), con estudios primarios (92\%) y ocupados laboralmente (50\%). La media de edad es de 37 años (rango 20-49 años) y el 77\% tiene estancias en prisión. Cuentan con una amplia historia de consumo de drogas (18 años de opiáceos, 15,8 de cocaína y 13,9 de benzodiacepinas), aunque llevan una media de 77 meses en programa de metadona (rango 13128). En la primera recogida de datos, se detectaban en orina restos de drogas en el 19\% de los casos para opiáceos, en el $35 \%$ para cocaína y en el $52 \%$ para benzodiacepinas.

La mayor parte se encuentra en el estadío B (44\%) y C (34\%) de la enfermedad, con una media de carga viral de 25.530 copias $/ \mathrm{ml}$, con el $45 \%$ de los pacientes con carga viral inferior a 50 copias $/ \mathrm{ml}$. La mayor parte $(52 \%)$ presenta entre 200 y 500 $\mathrm{CD}_{4}$, con una media de 398,7. Todos ellos habían tenido en algún momento de su vida una prescripción de fármacos antirretrovirales.

En el seguimiento se recogen únicamente datos de 91 personas. De los restantes, cuatro han fallecido y el resto han sido dados de alta en el programa de mantenimiento con metadona.

\section{Variables del estudio}

Las variables que se han incluido en este estudio son las siguientes:

\section{Variables de adhesión a la TARGA}

- Autoinforme sobre adhesión, con tres criterios temporales, ayer, la última semana y el último mes. Los valores inicialmente adoptados iban 
desde un cumplimiento perfecto, de todas las pastillas del día anterior, todos los días de la semana y del mes, a incumplimiento total con algunos grados intermedios. El tratamiento dado a la variable será dicotómico, por lo que consideraremos únicamente como adherentes a aquellos participantes que cumplan perfectamente con toda la medicación. El carácter longitudinal del estudio hará que finalmente podamos diferenciar distintos niveles de adhesión, y sobre todo, la evolución ocurrida con la misma. Así, tendremos finalmente cuatro grupos de participantes: adherentes que siguen siendo adherentes al cabo de año de seguimiento, adherentes que han abandonado el tratamiento, pacientes no adherentes en 2003 que han iniciado terapia antirretroviral y aquellos que siguen sin tomar la medicación.

\section{Variable psicosocial incluida en el estudio}

\section{- Apoyo Social percibido parejalfamilia y apoyo} percibido de los técnicos del CAID.

Encuesta elaborada ad hoc para evaluar el apoyo social percibido por el paciente sobre la familia, pareja y los técnicos del CAID/Hospital. Consta de cuatro ítems referidos, dos de ellos, al apoyo percibido para la ayuda a problemas del paciente y otros dos ítems a cómo se ha sentido apoyado en general por su familia y/o pareja y por los técnicos del CAID. Se ha utilizado una escala tipo Likert de (0) "Nunca" a (4) "Siempre" para los dos primeros ítems y de (0) "Ningún apoyo" a (4) "Muy apoyado" para los dos últimos ítems. Tras un análisis factorial se comprobó que la estructura de la escala era multifactorial, agrupándose los ítems referidos a la familia/amigos y los de los técnicos en factores independientes, por lo que se crearon dos sub-escalas. La de apoyo percibido por los pacientes desde los técnicos del CAID/Hospital presentó una consistencia interna de $\alpha=0,76$ para la primera recogida de datos y de $\alpha=0,72$ para la segunda y la subescala de apoyo percibido por los pacientes desde la familia/pareja presentó una consistencia interna de $\alpha=0,80$ para la primera recogida de datos y de $\alpha=0,77$ para la segunda.

\section{Procedimiento}

La recogida de datos se hizo entre los meses de Abril, Mayo y Junio de 2003 y en los mismos meses del año siguiente. Para la realización del estudio se tomó el listado de todos los usuarios que estaban en el programa de mantenimiento con metadona, y se seleccionaron aquellos pacientes que fueran VIH+. Se consultó la historia clínica para comprobar que en algún momento de su tratamiento de metadona en el CAID habían iniciado algún periodo de tratamiento con antirretrovirales, independientemente de que continuaran con él o no. Una vez recogidos estos datos se les citaba con el entrevistador y se les explicaban las características del estudio invitándoles a participar en él. Todos debían firmar el consentimiento informado y en el transcurso de esta misma cita se les pasaba el cuestionario de evaluación diseñado ad hoc para esta investigación. Las pruebas eran realizadas por el entrevistador de modo que se acortara el tiempo y que no cansara a los participantes, entre 15 y 20 minutos.

Una vez recogidos los datos, se tabularon con el programa estadístico SPSS para Windows, para su posterior análisis estadístico. La estrategia seguida en este caso ha sido analizar los cambios que presentan los participantes en función del tipo de adhesión que presentaban, sus cambios, y las diferencias encontradas en la variable apoyo social en función de la misma.

\section{Resultados}

En cuanto a la adhesión al tratamiento antirretroviral, en el año 2003 el $61 \%$ realizó el día anterior la toma correcta, mientras que en el año 2004 lo hizo el $69 \%$. Para el caso de la última semana, lo hicieron el $60 \%$ y $64 \%$, respectivamente y para el mes fueron el $44 \%$ y $52 \%$. Cuando se analizan los cambios ocurridos, encontramos que siguen siendo adherentes al cabo de un año el $51 \%$, el $47 \%$ y el $34,9 \%$, mientras que siguen sin tomar correctamente la medicación el 13,4\%, el 19,3\% y hasta el $36 \%$. Entre un 12 y un $18 \%$ de participantes cambian su condición para cada una de las categorías (tabla 1). 
Tabla 1. Frecuencias de adherencia y evolución entre el primer y el segundo momento de recogida de datos

Adherencia al tratamiento antirretroviral

\begin{tabular}{|c|c|c|c|c|}
\hline & & \multicolumn{3}{|c|}{ Momento 2} \\
\hline Ayer & \multicolumn{2}{|c|}{$61(61.0 \%)$} & \multicolumn{2}{|l|}{$58(69.0 \%)$} \\
\hline Semana & \multicolumn{2}{|c|}{$60(60.0 \%)$} & \multicolumn{2}{|l|}{$54(63.5 \%)$} \\
\hline Mes & \multicolumn{2}{|c|}{$44(44.0 \%)$} & \multicolumn{2}{|l|}{$44(51.8 \%)$} \\
\hline \multicolumn{5}{|c|}{ Cambios en adherencia } \\
\hline \multicolumn{2}{|r|}{$\begin{array}{c}\text { Cambio negativo, } \\
\text { abandona } \\
\text { el tratamiento }\end{array}$} & $\begin{array}{l}\text { Sigue mal, } \\
\text { sin tratamiento }\end{array}$ & $\begin{array}{l}\text { Cambio positivo, } \\
\text { inicia tratamiento }\end{array}$ & $\begin{array}{l}\text { Sigue bien, } \\
\text { adherente }\end{array}$ \\
\hline Ayer & $15(18.3 \%)$ & $11(13.4 \%)$ & $14(17.1 \%)$ & $42(51.2 \%)$ \\
\hline Semana & $15(18 \%)$ & $16(19.3 \%)$ & $13(15.7 \%)$ & $39(47 \%)$ \\
\hline Mes & $10(12 \%)$ & $30(36.1 \%)$ & $14(16.9 \%)$ & $29(34.9 \%)$ \\
\hline
\end{tabular}

En relación al apoyo social, podemos comprobar que la muestra percibe en el momento inicial un apoyo similar de la familia, media de 9,6 (desviación típica 3) y de los técnicos del CAID y del Hospital, media de 9,5 $(2,4)$, pero que ambos se reducen de manera estadísticamente significativa con el paso del tiempo, media $8,8(3,4)$ el apoyo familiar $(\mathrm{t}=2,12, \mathrm{p}=.037)$ y $8,6(2,4)$ para el apoyo del CAID/Hospital $(\mathrm{t}=3,41, \mathrm{p}<.000)$.

Además, ni el apoyo inicial ni la evolución a lo largo del tiempo son iguales en los cuatro grupos de adhesión. En la tabla 2 se recogen las compara- vas. En la tabla 3 se recogen las comparaciones por pares a partir de los contrastes no paramétricos con dos muestras independientes, utilizando la prueba de Mann-Whitney y aplicando la corrección de Bonferroni, lo que nos ha llevado a considerar significativas únicamente diferencias con una probabilidad inferior a 0.008 . Como se puede comprobar en la tabla, las diferencias más importantes se dan entre los particiapantes que siguen sin ser adherentes al cabo de un año frente a los que han seguido adherentes, siendo éstos últimos los que tienen más apoyo tanto familiar como técnico y conside-

Tabla 2. Diferencias en apoyo social según grupos de adherencia. Contrastes no paramétricos Kruskall-Wallis

\begin{tabular}{llccc}
\hline & & Chi-cuadrado & g.l & P. \\
\hline Apoyo familia/amigos & Ayer & 18.318 & 3 & .000 \\
& Semana & 15.997 & 3 & .001 \\
& Mes & 14.984 & 3 & .002 \\
\hline \multirow{2}{*}{ Apoyo CAID/Hospital } & Ayer & 21.342 & 3 & .000 \\
& Semana & 18.596 & 3 & .000 \\
& Mes & 16.347 & 3 & .001 \\
\hline
\end{tabular}

ciones entre los cuatro grupos aplicando la prueba de rangos (Kruskall-Wallis) para la primera medida de apoyo social. A modo indicativo de las magnitudes, las tablas 4 y 5 recogen las medias de cada uno de los grupos en apoyo social de la familia y de los técnicos del CAID y del hospital. Como se puede comprobar, en todos los casos se encuentra que los grupos difieren en sus niveles previos de apoyo. Ahora bien, una vez comprobado que se dan diferencias, lo revelante es encontrar entre qué pares de grupos son estadísticamente significati- rando los tres niveles de adhesión. Algo similar ocurre con los que hacen un cambio negativo, que muestran el mismo patrón, salvo para la adhesión mensual del apoyo de los técnicos del CAID. Dado que la diferencia inicial era que los que iban a hacer un cambio negativo eran adherentes en el momento de la primera medida podemos decir que las diferencias se dan entre pacientes adherentes y no adherentes. En esta misma línea se pueden interpretar las otras dos diferencias encontradas para la adhesión del día de ayer, los que hacen un 
Tabla 3. Diferencias en apoyo social según grupos de adherencia. Comparación por pares ${ }^{1}$

\begin{tabular}{|c|c|c|c|c|c|c|c|c|c|c|}
\hline \multicolumn{11}{|c|}{ Apoyo familia amigos } \\
\hline \multirow{2}{*}{$\begin{array}{c}\text { Grupo } \\
\text { adherencia }\end{array}$} & \multicolumn{3}{|c|}{ Ayer } & \multicolumn{3}{|c|}{ Semana } & \multicolumn{3}{|c|}{ Mes } & \multirow{2}{*}{$\begin{array}{c}\text { Grupo } \\
\text { adherencia }\end{array}$} \\
\hline & \multicolumn{2}{|c|}{ Mann-Whitney } & \multirow{2}{*}{$\begin{array}{c}\mathrm{P} \\
.002\end{array}$} & \multicolumn{2}{|c|}{ Mann-Whitney } & \multirow{2}{*}{$\begin{array}{c}\mathrm{P} \\
.001\end{array}$} & \multicolumn{2}{|c|}{ Mann-Whitney } & \multirow{2}{*}{$\begin{array}{c}\mathrm{P} \\
.002\end{array}$} & \\
\hline $\begin{array}{l}\text { Sigue mal } \\
\text { Sigue mal }\end{array}$ & $<$ & $\begin{array}{l}93.00 \\
=\end{array}$ & & $<$ & $\begin{array}{l}136.00 \\
=\end{array}$ & & $<$ & $\begin{array}{l}236.00 \\
=\end{array}$ & & $\begin{array}{c}\text { Sigue bien } \\
\text { Cambio positivo }\end{array}$ \\
\hline Sigue mal & \multirow[t]{3}{*}{$<$} & 19.00 & \multirow[t]{3}{*}{.001} & \multirow[t]{4}{*}{$<$} & 41.00 & \multirow[t]{4}{*}{.001} & \multirow[t]{4}{*}{$<$} & 68.50 & \multirow[t]{4}{*}{.009} & Cambio negativo \\
\hline Sigue bien & & $=$ & & & $=$ & & & $=$ & & Cambio positivo \\
\hline Sigue bien & & $=$ & & & $=$ & & & $=$ & & Cambio negativo \\
\hline Cambio positivo & $<$ & 41.00 & .004 & & $=$ & & & $=$ & & Cambio negativo \\
\hline & & & & \multicolumn{6}{|c|}{ Apoyo CAID/Hospital } & \\
\hline \multirow{6}{*}{$\begin{array}{l}\text { Sigue mal } \\
\text { Sigue mal } \\
\text { Sigue mal } \\
\text { Sigue bien } \\
\text { Sigue bien } \\
\text { Cambio positivo }\end{array}$} & \multirow[t]{2}{*}{$<$} & 69.00 & \multirow[t]{2}{*}{.000} & \multirow[t]{2}{*}{$<$} & 110.00 & \multirow[t]{2}{*}{.000} & \multirow[t]{6}{*}{$<$} & 192.00 & \multirow[t]{6}{*}{.000} & Sigue bien \\
\hline & & $=$ & & & $=$ & & & $=$ & & Cambio positivo \\
\hline & $<$ & 29.50 & .004 & \multirow[t]{4}{*}{$<$} & 53.00 & \multirow[t]{4}{*}{.007} & & $=$ & & Cambio negativo \\
\hline & $>$ & 119.50 & .001 & & $=$ & & & $=$ & & Cambio positivo \\
\hline & & $=$ & & & $=$ & & & $=$ & & Cambio negativo \\
\hline & & $=$ & & & $=$ & & & $=$ & & Cambio negativo \\
\hline
\end{tabular}

cambio positivo inicialmente tenían menos apoyo de la familia que los que hacen un cambio negativo, mientras que los que siguen bien cuentan con más apoyo técnico que los que hacen un cambio positivo.

Por lo que respecta a la evolución a lo largo del tiempo, las tabla 4 y 5 muestran la comparación de medias entre los dos momentos de medida para los dos tipos de apoyo y para los cuatro grupos de adhe- sión considerados en los tres momentos temporales, el día, la semana y el mes anterior a la encuesta. En cuanto al apoyo familiar (tabla 4), la evolución más llamativa se encuentra en los grupos que hacen cambios negativos (adhesión ayer, semana y mes). Las variaciones que se dan en el resto de casos, ligero incremento en los que cambian para bien o decremento en los que se mantienen sin tratamiento, no es significativo.

Tabla 4. Medias pre-post en los distintos grupos de adherencia. Apoyo Familia/amigos

\begin{tabular}{|c|c|c|c|c|c|c|c|}
\hline & & \multicolumn{2}{|c|}{ Medida 1} & \multicolumn{2}{|c|}{ Medida 2} & \multirow[t]{2}{*}{$\mathrm{T}$} & \multirow[t]{2}{*}{$\mathrm{P}$} \\
\hline & & Media & Desv. típ. & Media & Desv. típ. & & \\
\hline \multicolumn{8}{|c|}{ Adherencia ayer } \\
\hline Sigue mal (no adherente) & 10 & 7.20 & 3.88 & 6.10 & 2.84 & .974 & .356 \\
\hline Sigue bien (adherente) & 42 & 10.14 & 2.94 & 10.04 & 3.09 & .219 & .828 \\
\hline Cambio negativo & 15 & 11.00 & 1.88 & 6.93 & 3.59 & 5.4497 & .000 \\
\hline Cambio positivo & 14 & 8.64 & 2.67 & 9.92 & 2.26 & -1.312 & .212 \\
\hline \multicolumn{8}{|c|}{ Adherencia semana } \\
\hline Sigue mal (no adherente) & 15 & 7.40 & 3.52 & 6.66 & 2.58 & .889 & .389 \\
\hline Sigue bien (adherente) & 39 & 10.12 & 3.03 & 10.05 & 3.21 & .165 & .870 \\
\hline Cambio negativo & 15 & 10.80 & 1.93 & 6.86 & 3.54 & 5.059 & .000 \\
\hline Cambio positivo & 13 & 9.30 & 2.65 & 10.53 & 1.94 & -1.199 & .254 \\
\hline \multicolumn{8}{|c|}{ Adherencia mes } \\
\hline Sigue mal (no adherente) & 29 & 8.51 & 3.63 & 7.37 & 3.12 & 1.374 & .189 \\
\hline Sigue bien & 29 & 10.51 & 2.64 & 10.31 & 2.90 & .722 & .477 \\
\hline Cambio negativo & 10 & 10.90 & 2.28 & 7.40 & 4.01 & 3.748 & .005 \\
\hline Cambio positivo & 14 & 9.14 & 2.44 & 10.37 & 2.76 & -1.574 & .140 \\
\hline
\end{tabular}


Tabla 5. Medias pre-post en los distintos grupos de adherencia. Apoyo CAID/Hospital

\begin{tabular}{|c|c|c|c|c|c|c|c|}
\hline & & \multicolumn{2}{|c|}{ Medida 1} & \multicolumn{2}{|c|}{ Medida 2} & \multirow[t]{2}{*}{$\mathrm{T}$} & \multirow[t]{2}{*}{$\mathrm{P}$} \\
\hline & & Media & Desv. típ. & Media & Desv. típ. & & \\
\hline \multicolumn{8}{|c|}{ Adherencia ayer } \\
\hline Sigue mal (no adherente) & 10 & 7.50 & 2.50 & 5.40 & 2.41 & 2.973 & .016 \\
\hline Sigue bien (adherente) & 42 & 10.50 & 1.92 & 9.52 & 2.27 & 2.763 & .009 \\
\hline Cambio negativo & 15 & 10.13 & 2.16 & 7.73 & 2.71 & 3.674 & .003 \\
\hline Cambio positivo & 14 & 8.14 & 2.24 & 9.57 & 2.70 & -1.973 & .070 \\
\hline \multicolumn{8}{|c|}{ Adherencia semana } \\
\hline Sigue mal (no adherente) & 15 & 7.53 & 2.66 & 6.00 & 2.53 & 2.253 & .023 \\
\hline Sigue bien (adherente) & 39 & 10.51 & 1.97 & 9.53 & 2.34 & 2.595 & .013 \\
\hline Cambio negativo & 15 & 9.93 & 2.12 & 7.73 & 2.71 & 3.182 & .007 \\
\hline Cambio positivo & 13 & 8.92 & 2.06 & 10.30 & 1.79 & -1.793 & -098 \\
\hline \multicolumn{8}{|c|}{ Adherencia mes } \\
\hline Sigue mal (no adherente) & 29 & 8.48 & 2.51 & 7.17 & 2.66 & 3.007 & .006 \\
\hline Sigue bien & 29 & 10.75 & 1.78 & 9.58 & 2.27 & 2.745 & .010 \\
\hline Cambio negativo & 10 & 10.30 & 2.26 & 8.50 & 3.02 & 1.784 & .108 \\
\hline Cambio positivo & 14 & 9.07 & 2.30 & 10.07 & 2.52 & -1.242 & .236 \\
\hline
\end{tabular}

En el apoyo profesional, del CAID y del hospital, se encuentran más variaciones (tabla 5). Inicialmente se observa que aquellos pacientes que siguen sin ser adherentes (ayer, semana y mes) perciben una reducción del apoyo, lo mismo que les ocurre a los que hacen un cambio negativo. Por su parte, los que siguen bien, perciben igualmente que su apoyo técnico se va reduciendo. Por último, las mejorías percibidas por los que hacen un cambio positivo no llegan a ser estadísticamente significativas.

\section{Discusión}

Una de las primeras conclusiones que se puede extraer de nuestro estudio es que la adhesión cambia con el transcurso del tiempo. Los datos reflejan que para la adhesión mensual los valores son menores que para la adhesión semanal o del día anterior, sin embargo, para todas las categorías de adhesión consideradas se observa un aumento de la misma con el paso del tiempo, siendo cada vez mayor el número de pacientes que informan de adhesión correcta. Estos resultados no coinciden con los hallados por Howar et al (2002) quienes, en su estudio prospectivo multicéntrico, encuentran una disminución del $10 \%$ en el nivel de adhesión con el paso del tiempo.
Otro resultado encontrado en referencia a la variable percepción de apoyo social es que se encuentra relacionada con la adhesión a la medicación antirretroviral y que sufre variaciones durante el transcurso del año evaluado. Los pacientes perciben en general una pérdida significativa del apoyo social, tanto de su familia como de los equipos técnicos de los que reciben asistencia sanitaria, siendo esta pérdida diferente en cada uno de los grupos contemplados. Todos los grupos difieren en sus niveles previos de apoyo social en el primer año evaluado y también presentan distintos niveles de percepción de apoyo social en el segundo año. Asumiendo esta diferencia en el nivel de apoyo social percibido en el inicio y el final de la evaluación, los pacientes que perciben una mayor pérdida de apoyo social son aquellos que permanecen no adherentes con el paso del tiempo. No sabemos si este dato responde al fracaso personal por no seguir la medicación, o por el contrario, aparece asociado a otras circunstancias como el consumo de drogas, presente en una parte de nuestra muestra o a la negativa a tomar la medicación por no creer en ella, no tolerar los efectos secundarios o haber tenido intentos anteriores poco exitosos, lo que ha podido mermar su autoeficacia, otro factor que influye en la adhesión (Wilson et al., 2004; Ladero et al., 2005). En cualquier caso, la baja percepción de apoyo 
social de estos pacientes y el no tomar el tratamiento antirretroviral les pone en una situación de doble riesgo, sobre todo teniendo en cuenta que a medida que se progresa en la enfermedad factores psicológicos como la depresión se convierten en pronósticos de una más rápida evolución de la misma (Cook et al., 2004).

También, los participantes que se incorporan a tomar el tratamiento antirretroviral durante el año evaluado parten de una menor percepción de apoyo familiar en el inicio que los que abandonan el tratamiento, que a su vez, lo ven mermado con el paso del tiempo. Esto podría ser explicado, en parte, por la posibilidad que a los pacientes que llevan a cabo un cambio positivo les brinda su entorno familiar de comenzar y mantener el tratamiento de desintoxicación/deshabituación de drogas, de iniciar y/o controlar la toma de la medicación antirretroviral, así como de las expectativas positivas generadas al afrontar de manera exitosa la enfermedad, mientras que a los pacientes que abandonan el tratamiento les puede ocurrir exactamente lo contrario: retirada de apoyo familiar por abandonar el tratamiento de deshabituación de drogas, recaída en el consumo de drogas, etc. Por otra parte, no podemos dejar de mencionar otros factores que también se han encontrado importantes en otros trabajos del mismo grupo de autores y que podrían incidir en el abandono de los tratamientos antirretrovirales de estos pacientes, tales como los estados emocionales negativos, la autoeficacia percibida, el esfuerzo percibido, etc (Chen, 2006; Gordillo, Del Amo, Soriano y Gonzalez-Lahoz ，1999; Gordillo, 2003; Ladero, Orejudo y Carrobles, 2005; Liu et al., 2006).

Por último, en cuanto a la evolución experimentada del apoyo social percibido desde los equipos técnicos del CAID y del hospital, resaltar que éste se ve disminuido con el paso del tiempo entre aquellos participantes que siguen sin ser adherentes, los que abandonan la medicación y los que siguen bien con su tratamiento antirretroviral. Esto último podría indicar que los pacientes que siguen bien con su tratamiento antirretroviral necesitarían menor apoyo técnico que el resto de los grupos, ya que podrían haber experimentado cierto grado de generalización en el aprendizaje del cuidado de su salud: cierta autonomía en la adhesión a la TARGA, visitas a revisiones hospitalarias, así como en la abstinencia a drogas, etc. La explicación contraria sería plausible sin embargo, para los grupos de pacientes que siguen sin ser adherentes y los que abandonan la medicación, en el que el abandono del tratamiento antirretroviral o el no inicio del mismo coincidiría con una menor necesidad de solicitar ayuda a los técnicos sanitarios. Esta explicación viene avalada por el hecho de que el grupo de participantes que se incorporan a tomar la medicación antirretroviral en el transcurso del año evaluado experimentan un aumento en la percepción de apoyo técnico, aunque este aumento no resulte estadísticamente significativo.

Todo lo anterior podría indicar que en el caso de los pacientes con antecedentes de consumo de drogas el inicio de sus tratamientos antirretrovirales coinciden en el tiempo con el comienzo de los programas de deshabituación de drogas, aunque posteriormente pueden darse abandonos de ambos programas (Muga et al., 2004). Así, los drogodependientes que se sienten más apoyados por los profesionales de los centros de atención a las drogodependencias, con menores barreras de acceso a los recursos y que se sienten más apoyados emocionalmente por sus familiares y/o amigos tendrían mayor probabilidad de conseguir abstinencia que aquellos que carezcan de este apoyo (Davis, 2008; Skeie et al., 2007).

La conclusión que se puede extraer de los datos aquí analizados es que hay una clara relación entre el apoyo social percibido y la adhesión a la terapia antirretroviral en pacientes en programas de mantenimiento con metadona (Kidorf et al., 2005). Esta hipótesis vendría reforzada por el hecho de que las diferentes intervenciones diseñadas para aumentar la adhesión muestran cómo la consecución de apoyo familiar y la eliminación de barreras de acceso a los tratamientos médicos de control de VIH/SIDA consigue aumentar la adhesión a los tratamientos antirretrovirales (Shelton et al., 2006; Ballester, 2003 y 2005; Koening et al., 2008). La variable apoyo social percibido, al igual que otras consideradas en el estudio, como la autoeficacia, el consumo de drogas, los estados emocionales negativos, sin olvidar otros aspectos como las dificultades propias del régimen o aspectos farmacológicos como toxicidad, 
interacciones con otros fármacos, etc; pueden ayudar a entender un comportamiento tan complejo como es la adhesión a la TARGA.

No quisiéramos acabar sin señalar algunas de las limitaciones de este trabajo, quizás la más importante de ella es haber considerado una sola variable con relación a la adherencia cuando se sabe que inciden varios tipos de factores en la adhesión a la TARGA en esta población. A pesar de que esto es así y que ya en otros trabajos hemos analizado el papel que otras variables tienen en la adherencia al tratamiento antirretroviral en la población usuaria de drogas (Ladero, Orejudo y Carrobles, 2005; Ladero, Orejudo y Carrobles, 2008; Orejudo, Ladero, Carrobles y Malo, 2006), entendemos que analizar el papel del apoyo social de manera independiente del resto permite destacar la relevancia de esta variable, más aún cuando es posible su modificación como una claro medio para la mejora de los pacientes.

\section{Extended Summary}

In Spain, the most affected group from HIV/Aids is those formed by by parenteral administration drug users. Opiate substitution treatments, such as methadone, together with treatments for HIV/Aids (High Activity Anti Retroviral Therapy, or HAART) have contributed to increasing the life expectancy in this population (Clarke et al., 2003). Nonetheless, adherence to the different treatments continues to be one of the main problems that make therapeutic achievement difficult from these treatments. There are many and varied factors to be considered that could be influencing adherence. Drug consumption has been demonstrated to be one of the factors that can negatively influence adherence to HAART (Battaglioli- DeNero, 2007). Likewise, social support could be considered as a factor that influences positively both the adhesion to the treatments for quitting drug use as well as antiretroviral treatments. It has been proven that psychosocial intervention improves the effectiveness of the methadone treatment, just as family and technical support do (Préau et al., 2006). Better results in adhesion to HAART is also seen in those patients who find no barriers to the access of clinics, who have good communication with professionals and who feel more supported emotionally at the beginning of and during the treatment (Knowlton et al., 2006).

This study seeks to explore the relationships and variations experienced over a period of a year, between the adhesion to antiretroviral treatment and social support in HIV patients in methadone treatment programs. The main objective would be to better understand the evolution of both variables to increase the efficiency of HAART. It is about find- ing empirical support for this relationship and establishing if social support can be considered as a precedent variable or a consequence of adherence. Firstly, data from $100 \mathrm{HIV}+$ patients was taken randomly when they went to pick up their methadone treatment. They were all over 18 years of age, HIV positive and at some point had had anti retroviral prescriptions. Most of them were male (80\%), of a low social-economic level $(71 \%)$, with elementary studies (92\%) and working (50\%), with an average of 37 years old and a long history of drug consumption. Indeed, in the first data collection, through urine exams, drug rests were detected in $19 \%$ of the cases for opiates, $35 \%$ cocaine and $52 \%$ benzodiazepines. Their health state was diverse; most of them were in Stages B (44\%) and C (34\%) of the illness, with an average viral count of 25.530 copies $/ \mathrm{ml}$, with $45 \%$ of the patients having a viral count lower than 50 copies $/ \mathrm{ml}$. The majority (52\%) present between 200 and 500 CD4 with an average of 398,7 .

A year later they were contacted again, locating only 83 persons (four had passed away, five were discharged from the methadone program and another 8 could not be reached) and their evolution since then was compared. They were classified in four different groups based on their evolution that year: patients that were never adherent and continued not to be, patients that were adherent and still were and patients that changed their adhesion, they either abandoned the treatment or they began the treatment. This classification was made taking three different indicators of adhesion according to a self report by the patients, about their taking of medica- 
tion the day, the week and the month prior to the interview.

Together with adhesion, the other variable included in the study was social support, which was evaluated both at the beginning as well as in the following year. For this, an instrument was used with four items elaborated ad hoc (Social Support perceived from their partner/family and social support perceived from the CAID technicians) (the CAID refers to the Centers for Attention to Drug Dependants), which includes perceived support both from the family as well as from professionals (hospital and centers for attention to drug dependants). The internal consistency of the instruments varies between 0,72 and 0,80 in the two data collections.

The results obtained demonstrated changes in adhesion. Thus, $51 \%$ continued to be adherent (the day before), $47 \%$ (the week before) and $34,9 \%$ (the month before), while $13,4 \%, 19,3 \%$ and up to $36 \%$ continued not to take their medication correctly. On the other hand, $17,1 \%, 15,7 \%$ and $16,9 \%$ respectively made a positive change, this is, they began the treatment. On the contrary, $18,3 \%, 18 \%$ and $12 \%$ abandoned it. With regards to social support, the results found demonstrate that the group perceived at the beginning a similar support from family $(9,55)$ and from the CAID Center's and hospital technicians $(9,54)$, but this began to reduce statistically significantly through time, in the first case to 8,78 and in the second to 8,57 . Nonetheless, the most relevant finding is that neither the initial social support nor the evolution with time is the same in the four adherence groups mentioned.

As for the levels of previous support, the most important differences are given between the patients that continue not being adherent and those that continue being adherent, with those that remain non adherent being the ones that perceive a lesser social support, while those that continue being adherent have more support both from their families as well as from the technicians for all the categories considered (adhesion the day, week and month before). Those that make a negative change show the same pattern as those that remain non adherent, except for the monthly adhesion from the support of the CAID Center. Given that the initial difference was that those who were going to make a negative change were not adherent at the time of the first measure, we can say that the differences are found between adherent patients and non adherent ones. The other two differences found for adhesion on the day before can be interpreted in a similar way. Those that make a positive change initially had less family support than those that made a negative change, while those that continue well have more technical support than those that made a positive change. We do not know if the fact that the non adherent patients suffer a major loss of social support is a response to the personal failure of not following the medication, or appears associated to circumstances like drug use, present in a part of our sample group, or negatively to taking the medication but not believing in it, not tolerating the side effects or having had prior attempts that were not very successful, which could have reduced the self efficacy, another factor which influences adhesion (Wilson et al., 2004; Ladero et al., 2005). In either case, the low perception of social support of these patients and the not taking the antiretroviral treatment puts them in a situation of double risk, particularly considering that as the illness progresses, psychological factors such as depression become a prognosis of a faster evolution of the illness (Cook et al., 2004).

As for the evolution in the long term and of family support, the most striking change is found in the group that makes a negative change, for whom statistically significant decreases are found, while there are barely any variations in the rest of the groups. As we have already mentioned, the group which began taking the antiretroviral medication during the year came from a lower perception of family support in the beginning than those who abandoned the treatment, which also saw it reduced with the passing of the time. This could be explained in part by the possibility that for the patients who undergo a positive change, their family support offers them to begin and continue treatment of drug detoxification, to begin and/or control their antiretroviral medication intake, as well as the positive expectations generated while successfully confronting the illness, while for the patients who abandon the treatment the exact opposite can occur.

As for the professional support both from the CAID Center and the hospital, we found more vari- 
ations, seeing that the patients that continue being adherent perceive a reduction in the support. The same occurs to those that make a negative change. On the other hand, the improvements perceived by those who make a positive change are not statistically significant and those who continue well also perceive that their technical support is reducing. This last piece of information could indicate that the patients who continue well with their antiretroviral treatment would need less technical support than the rest of the groups, since they could have experienced a certain level of generalization in learning about their health care: certain autonomy from the adhesion of the HAART, hospital checkup visits, drug abstinence, etc. The opposite explanation could be plausible for the groups of patients that continue not being adherent and those that abandon the treatment. This explanation is endorsed by the fact that the group that starts taking the medication during the year evaluated experiences an increase in perception of technical support, even thought this increase is not significant.

Everything previously mentioned could indicate that in the case of patients with drug consumption history the beginning of their antiretroviral treatment coincides in time with the beginning of the drug detoxification programs, even if afterwards they can abandon both programs (Muga et al., 2004). Thus, the drug addicts that feel the most supported by professionals in drug addiction health centers, with fewer barriers to accessing resources and who feel the most supported by their families and/or friends would have a higher probability of achieving abstinence that those that lack this support (Skeie et al., 2007).

The final conclusion that can be drawn from the data analyzed here is that there is a clear relationship between perceived social support and adhesion to antiretroviral therapy in patients in methadone maintenance programs (Kidorf et al., 2005). Nonetheless, it is not easy to assume which variable acts as an antecedent of the other, since in the time interval analyzed, most changes occur together. This hypothesis would be reinforced by the fact that the different interventions designed to increase adhesion demonstrate how the achievement of family support and the elimination of barriers to the access of med- ical treatments for control of HIV/AIDS continues to increase the adhesion to antiretroviral programs (Ballester, 2003); Koening et al., 2008). Finally, we want to mention that there are other variables that can influence adhesion, such as drug consumption or emotional states, and social support could also have a direct relationship on these, or interact with them, all of which leaves, in any case, numerous questions pending in this field.

\section{Referencias}

Battaglioli-DeNero, A.M. (2007). Strategies for improving patient adherence to therapy and longterm patient outcomes. The Journal of the Association of Nurses in AIDS Care, 18 (1 Suppl), 17-22.

Ballester, R. (2002) • ¿Existe un patrón diferencial de conducta de enfermedad y adhesión al tratamiento de la infección por VIH/SIDA en pacientes con historia de abuso de drogas?. Revista de Psicopatología y Psicología Clínica, 8, 127-138.

Ballester, R. (2003). Eficacia terapéutica de un programa de intervención grupal cognitivo-comportamental para mejorar la adhesión al tratamiento y el estado emocional de pacientes con Infección por VIH/SIDA. Psicothema, 15, 517-523.

Ballester, R. (2005). Aportaciones desde la psicología al tratamiento de las personas con infección por VIH/SIDA. Revista de Psicopatología y Psicología Clínica, 10, 53-69.

Carballo, E., Cadarso-Suárez, C., Carrera, I., Fraga, J., De la Fuente, J., Ocampo, A., Ojea, R. y Prieto, A. (2004). Assessing relationships between health-related quality of life and adherence to antiretroviral therapy. Quality of Life Research: An International Journal of Quality of Life Apects of Treatment, Care \& Rehabilitation, 13, 587599.

Carrobles, J.A., Remor, E.A. y Rodríguez-Alzamora, L. (2003). Afrontamiento, apoyo social percibido y distrés emocional en pacientes con infección por VIH, Psicothema, 15, 420-426

Clarke, S., Delamere, S., McCullough, L., Hopkins, S., Bergin, C. y Mulcahy, F. (2003). Assessing limiting factors to the acceptance of antirretrovi- 
ral therapy in a large cohort of injecting drug users. HIV Medicine, 4, 33-37.

Cook, J.A., Grey, D., Burke, J, Cohen, M.H., Gurtman, A.C., Richardson, J.L., Wilson, T.E., Young, M, y Hessol, N.A. (2004). Depressive Symptoms and AIDS-related mortality among a multisite cohort of HIV-positive women. American Journal of Public Health, 94, 11331140.

Costenbader, E.C., Astone, N.M.y Latkin, C.A. (2006). The dynamics of injection drug users' personal networks and HIV risk behaviors. Addiction, 101, 1003-1013.

Chen, G. (2006). Social support, spiritual program, and addiction recovery. International Journal of Offender Therapy and Comparative Criminology, 50, 306-23.

Dannerbeck, A., Harris, G., Sundet, P. y Lloyd, K. (2006). Understanding and responding to racial differences in drug court outcomes. Journal of Ethnicity in Substance Abuse, 5, 1-22.

Davis, E. M. (2008). Gendered issues in alcohol abuse and dependence among HIV-positive African Americans. Dissertation Abstracts International Section A: Humanities and Social Sciences, 69, 377.

Garrity, T.F., Prewitt, S.H., Joosen, M., Tindall, M.S., Webster, J.M., Hiller, M.L. y Leukefeld, C.G. (2006). Correlates of subjective stress among drug court clients. International Journal of Offender Therapy and Comparative Criminology, 50, 269-79.

Goodkin, K., Shapshak, P., Asthana, D., Zheng, W., Concha, M., Wilkie, F., Molina, R., et at. (2004). Older age and plasma viral load in HIV-1 infection. AIDS. Special Issue: HIVIAIDS and Aging, 18 (Suppl1), 587-598.

Gordillo, V., Del Amo, J., Soriano, V. y GonzalezLahoz, J. (1999). Sociodemografic and psychological variables influencing adherence to antiretroviral therapy. AIDS, 13, 1.763-1.769.

Gordillo, V. y de la Cruz, J. J. (2003). Adherencia y fallo terapeutico en el seguimiento de una muestra de sujetos VIH+: algunas hipotesis desde la psicologia. Psicothema, 15, 227-233.

Gustafsson, M. y Ahlström, G. (2006). Emotional distress and coping in the early stage of recovery following acute traumatic hand injury: a questionnaire survey. International Journal of Nursing Studies, 43, 557-65.

Hill, Z., Kendall, C. y Fernández, M. (2003). Patterns of Adherence to Antirretrovirals: Why Adherence has no simple. AIDS Patient Care \& STDs, 17, 519-525.

Hinkin, C.H., Barclay, T.R., Castellon, S.A., Levine, A.J., Durvasula, R.S., Marion, S.D., Myers, H.F. y Longshore, D. (2007). Drug use and medication adherence among HIV-1 infected individuals. AIDS and Behavior, 11, 185-94.

Howar, A., Arnsten, J., Lo, Y., Vlahov, D., Rich, J., Schuman, P., Stone, V., Smith, et al. (2002) A prospective study of adherence and viral load in a large multicenter cohort of HIV-infected women. AIDS, 16, 2175-2182.

Johnson, M.O., Chesney, M.A., Goldstein, R.B., Remien, R.H., Catz, S., Gore-Felton, C., Charlebois, E. y Morin, S.F. (2006). Positive provider interactions, adherence self-efficacy, and adherence to antiretroviral medications among HIV-infected adults: A mediation model. AIDS Patient Care STDS, 20, 258-68.

Kerr, T., Marshall, A., Walsh, J., Palepu, A., Tyndall, M., Montaner, J., Hogg, R. y Wood, E. (2005). Determinants of HAART discontinuation among injection drug users. AIDS Care, 17, 539-549.

Kidorf, M., King, V.L., Neufeld, K., Stoller, K.B., Peirce, J. y Brooner, R.K. (2005). Involving significant others in the care of opioid-dependent patients receiving methadone. Journal of Substance Abuse Treatment, 29, 19-27.

Knowlton, A., Arnsten, J., Eldred, L., Wilkinson, J., Gourevitch, M., Shade, S., Dowling, K. y Purcell, D. (2006). Individual, interpersonal, and structural correlates of effective HAART use among urban active injection drug users. Journal of Acquired Immune Deficiency Syndromes, 41, 486-92.

Koening, L., Pals, S., Bush, L., Pratt, T., Stratford, D., Ellerbrock, T.(2008). Randomized controlled trial of an intervention to prevent adherence failure among HIV-infected patients initiating antiretroviral therapy. Health Psichology, 27, 486-92.

Ladero, L., Orejudo, S.y Carrobles, J.A. (2005). Variables psicosociales en la adherencia al trata- 
miento antirretroviral en pacientes adscritos a un programa de mantenimiento con metadona. Psicothema, 17, 573-79.

Ladero, L., Orejudo, S. y Carrobles, J.A. (2008). Autoeficacia, esfuerzo y expectativas de control en la adhesión al tratamiento antirretroviral en pacientes VIH+ en un programa de mantenimiento con metadona. Estudio Longitudinal. Salud y Drogas 8, 73-92.

Liu, C., Johnson, L., Ostrow, D., Silvestre, A., Visscher, B.y Jacobson, L.P., (2006). Predictors for lower quality of life in the HAART era among HIV infected men. Journal of Acquired Immune Deficiency Syndromes, 42, 470-7.

Mino, M., Deren, S. y Yeon-Kang, S. (2006). Social support and HIV-related injection risk among Puerto Rican migrant and nonmigrant injection drug users recruited in New York City. AIDS Education and Prevention, 18, 81-90.

Maisels, L., Steinberg, J. y Tobias, C. (2001). An investigation of Why eligible patients do not receive HAART. AIDS Patient Care and STDS, 15, 185-91.

Muga, R., Egea, J.M., Sanvisens, A., Arnal, J., Tural, C. Tor, J y Rey-Joly, C. (2004). Impact of injecting drug use on the interruption of antirretroviral therapies. Journal of Epidemiology \& Community Health, 58, 286-287.

Orejudo, S., Ladero, L., Carrobles, J.A. y Malo Aznar, C. (2006). Ansiedad, depresión, estrés y adherencia al tratamiento antirretroviral en sujetos en un programa de mantenimiento con metadona. Ansiedad y Estrés, 12, 19-30.

Préau, M., Protopopescu, C., Spire, B., Dellamonica, P., Poizot-Martin, I., Villes, V.y Carrieri, M.P. (2006). Health related quality of life among HIV-HCV co-infected patients. Revue d'épidémiologie et de santé publique, 54 Spec No 1, 33-43.
Remor, E.A. (2002). Valoración de la adhesión al tratamiento antirretroviral en pacientes VIH+. Psicothema, 14 , 262-267

Reynolds N.R, Testa M.A, Marc L.G, Chesney M.A, Neidig J.L, Smith S.R, et al. (2004). Factors influencing medication adherence beliefs and self-efficacy in persons naïve to antiretroviral therapy: a multicenter, cross-sectional study. AIDS and Behavior, 8, 141-150.

Scherbaum, N., Kluwig, J., Specka, M., Krause, D., Merget, B., Finkbeiner, T. y Gaspar, M. (2005). Group Psychotherapy for opiate addicts in methadone maintenance treatment- A controlled trial. European Addiction Research, 11, 163-171.

Shelton, R.C., Golin, C.E., Smith, S.R., Eng, E. y Kaplan, A. (2006). Role of the HIV/AIDS case manager: analysis of a case management adherence training and coordination program in North Carolina. AIDS Patient Care STDS, 20, 193-204.

Skeie, I., Brekke, M., Lindbaek, M. y Waal, H. (2007). General practitioners can take responsibility for medication-based rehabilitation. Tidsskr Nor Laegeforen, 127, 296-297.

Spire, B., Duran, S., Souville, M., Leport, C., Raffi, F. y Moatti, J.P. (2002). Adherence to highly active antiretroviral therapies (HAART) in HIVinfected patients: Fron a predictive to a dynamic approach. Social Science \& Medicine, 54, 14811496.

Waddell, E.N. y Messeri, P.A. (2006). Social support, disclosure, and use of antiretroviral therapy. AIDS and Behavior, 10, 263-72.

Wilson, K.J., Doxanakis, A. y Fairley C.K. (2004). Predictors for non-adherence to antiretroviral therapy. Sexual Health, 1, 251-7.

Vlahov, D. y Celentano, D.D. (2006). Access to Highly Active Antiretroviral Therapy for inyection drug users: Adherence, resistence and death. Cadernos de saúde pública, 22, 705-731.

Artículo recibido: 26/02/2009

Revisión recibida: 06/03/2009

Aceptado: 18/03/2009 